\title{
Recognition and Parameter Extraction of One-Dimensional Electronic Scanning for 3D Radar
}

\author{
Cheng Li, ${ }^{1,2}$ Wei Wang, ${ }^{1,2}$ Longfei Shi, ${ }^{1,2}$ and Xuesong Wang ${ }^{2,3}$ \\ ${ }^{1}$ College of Electronic Science and Engineering, National University of Defense Technology, Changsha 410073, China \\ ${ }^{2}$ State Key Laboratory of Complex Electromagnetic Environmental Effects on Electronics and Information System, \\ National University of Defense Technology, Changsha 410073, China \\ ${ }^{3}$ College of Science, National University of Defense Technology, Changsha 410073, China
}

Correspondence should be addressed to Cheng Li; lchnudt@gmail.com

Received 15 May 2014; Revised 5 August 2014; Accepted 6 August 2014; Published 25 December 2014

Academic Editor: Matteo Pastorino

Copyright (C) 2014 Cheng Li et al. This is an open access article distributed under the Creative Commons Attribution License, which permits unrestricted use, distribution, and reproduction in any medium, provided the original work is properly cited.

\begin{abstract}
Most of the existing antenna scan type (AST) identification techniques focus on only mechanical scanning (MS) and rarely research electronic scanning (ES), especially one-dimensional (1D) ES. This paper proposes a recognition and parameter extraction method of 1D ES for 3D radar, which employs both MS and ES. An ES pattern simulator is designed to synthesize pulse amplitude (PA) data first. Then ID ES is distinguished from MS and two-dimensional (2D) ES based on the features extracted from the difference sequences of beam sequence, such as resemblance coefficient (RC) and variance. Subsequently, two vital parameters for 1D ES are extracted, namely, beam position number in elevation and pulse number in a beam position. Finally, some simulation results are shown to validate the proposed algorithm.
\end{abstract}

\section{Introduction}

In modern electronic warfare (EW), 3D radar [1-3] with electronically scanned beam in elevation, namely, onedimensional (1D) electronic scanning (ES), has been widely used for tactical applications. With the character of inertialess quick beam steering, active electronically scanned array [4] allows 3D radar to provide elevation information with range and azimuth simultaneously. Meanwhile, 3D radar brings a new set of challenges for electronic countermeasure (ECM). It is inadequate for electronic intelligence (ELINT) [5] and electronic support measurement (ESM) systems to obtain radar information based on the conventional parameters [6], such as radio frequency (RF), angle of arrival (AOA), time of arrival (TOA), pulse width (PW), pulse amplitude (PA), and pulse repetition interval (PRI). On the other hand, it is notoriously difficult to jam 3D radar by track deception [7]. Therefore, it is necessary to analyze some distinctive characters of 3D radar, for example, $1 \mathrm{D}$ ES.

The characteristic parameters of 1D ES, including antenna scan type (AST), antenna scan period (ASP), and some associated scanning parameters, are vital for EW systems.
From those parameters we can infer the antenna scanning technique, recognize the radar type, and even determine the threaten level. Despite the importance, there is a lack of studies in the open literature on $1 \mathrm{D}$ ES recognition and parameter extraction. The only related works mainly focus on the AST of mechanical scanning (MS) radar. In patents [8-10], various antenna scan pattern generators for MS radar simulation are invented. Literature [11] proposes a novel radar signal model which is able to generate radar signals for various scan patterns. In patent [12], Greer presents an automatic recognition method based on Laplace transform and fast Fourier transform (FFT) for basic ASTs. However, the variation of ASP and other parameters is neglected in the algorithm. In literature [13], Barshan and Eravci provide a novel ASP estimation and AST classification technique based on pattern recognition. Their algorithm seems effective and robust for 5 basic ASTs: circular scan, sector scan, raster scan, helical scan, and conical scan. However their studies do not take into account the ES.

The most obvious difference between MS and ES is that the latter changes the beam direction inertialessly and 
quickly. Since 3D radar employs both MS and ES, it is more complex to analyze its characters. To the best of our knowledge, the research on $1 \mathrm{D}$ ES identification and parameter extraction has remained elusive. The main contribution of this paper is providing lead recognition and parameter extraction of $1 \mathrm{D}$ ES for $3 \mathrm{D}$ radar. Firstly, we introduce the resemblance coefficient (RC) of the first- and second-order difference sequences as an efficient feature to distinguish ES from MS. Secondly, we give an approach to extract key parameters of $1 \mathrm{D}$ ES for $3 \mathrm{D}$ radar, including beam position number in elevation and pulse number in a beam position. Furthermore, we also make some improvements in the paper, such as the beam sequence extraction procedure. Many ELINT and ESM systems may benefit from our recognition of $1 \mathrm{D}$ ES. Furthermore, the parameters extracted in this paper, including ASP, beam position number in elevation, and pulse number in a beam position, are crucial for estimating the beam direction of $3 \mathrm{D}$ radar.

The rest of this paper is organized as follows. Section 2 provides some fundamental models and briefly describes the ES pattern simulator to synthesize PA data. In Section 3, the methodology for recognition and parameter extraction of $1 D$ ES is explained in detail. Some simulation results are presented in Section 4. Finally, the conclusion follows.

\section{Model and Simulator}

EW receiver detects radar parameters to acquire radar information, such as RF, AOA, TOA, PW, and PA. For antenna scanning analysis, time-domain analysis techniques based on measured PA data are commonly introduced. There is lack of recorded EW receiver data because of the classified nature of EW work. Therefore, it is necessary to simulate the received data based on signal models, especially PA data.

PA is the received pulse signal power of the EW antenna and is given by

$$
P_{r}(t)=\frac{P_{t} G_{r} G_{t} \lambda^{2}}{(4 \pi R)^{2} L} F_{t}[\theta(t), \phi(t)]
$$

where $P_{r}$ and $P_{t}$ are the received and the transmitted power, respectively, $G_{r}$ and $G_{t}$ are the antenna gains of EW receiver and transmitter, respectively, $\lambda$ is the wavelength, $R$ is the distance between the radar and the EW receiver, $L$ is the propagation loss factor, and $F_{t}[\theta(t), \phi(t)]$ is the transmitter antenna pattern at the offset angels $[\theta(t), \phi(t)]$, where the receiver is located at time $t$ and $\theta(t)$ and $\phi(t)$ are the azimuth angle and the elevation angle, respectively.

For the sake of simplicity, we assume that the radar and the EW receiver both remain stationary, the receiver antenna is isotropic, and the propagation loss is constant. As a result, the term $P_{t} G_{r} G_{t} \lambda^{2} /(4 \pi R)^{2} L$ is assumed to be constant, and the PA is mainly determined by $F_{t}[\theta(t), \phi(t)]$.

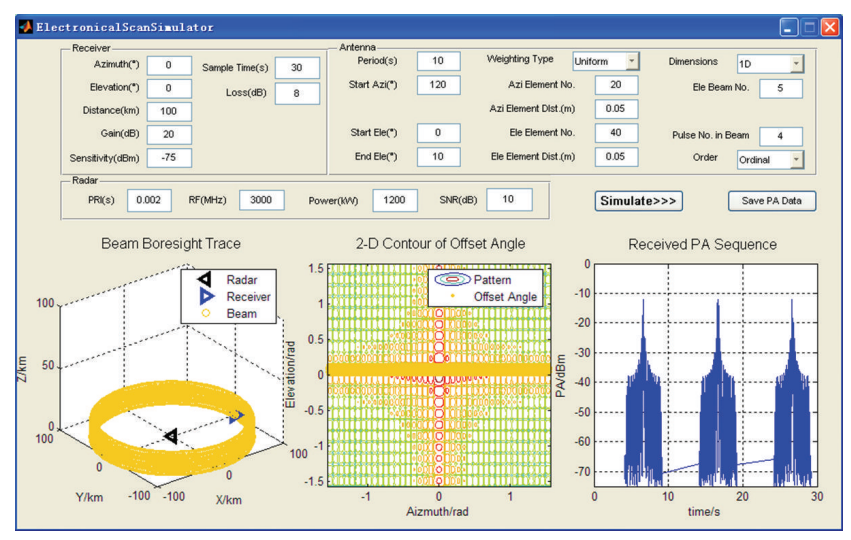

FIGURE 1: An example screen of the ES pattern simulator.

The transmitter antenna of the 3D radar is modeled as a planar array antenna in this paper. For a planar array antenna with $M \times N$ elements, the antenna pattern is given by [14]

$$
\begin{aligned}
& F(\theta, \varphi) \\
& =\sum_{m=0}^{M-1 N-1} \sum_{n=0} I_{m n} e^{j k\left[m d_{x}\left(\sin \theta \cos \varphi-\sin \theta_{0} \cos \varphi_{0}\right)+n d_{y}\left(\sin \theta \sin \varphi-\sin \theta_{0} \sin \varphi_{0}\right)\right]},
\end{aligned}
$$

where $I_{m n}$ is the $(m, n)$ element of the array weighting matrix $I_{M \times N}, d_{x}$ and $d_{y}$ are the element spacing along $x$ - and $y$ directions, respectively, $\theta_{0}$ and $\phi_{0}$ are the elevation angle and azimuth angle of beam, respectively, and $k=2 \pi / \lambda$.

In this paper, an ES pattern simulator with a graphical user interface (GUI) is developed using MATLAB [15]. An example screen of the simulator is illustrated in Figure 1, where the parameter setting part is located on the upper side, and 3 subgraphs are shown on the lower side, including the beam boresight trace, the $2 \mathrm{D}$ contour of offset angle, and the received PA versus TOA plot.

By the simulator, the ES antenna with optional weightings (uniform, Hamming, Hanning, Taylor, Kaiser, and Blackman) and different scanning parameters (start/end azimuth/elevation angles, azimuth/elevation element number, azimuth/elevation element distance, beam position number in elevation and pulse number in a beam position, etc.) can be simulated.

\section{Methodology}

The key step of the methodology in this paper is to recognize $1 D$ ES. As mentioned above, the measured PAs change continuously because of the inertial movement of beam for MS. For ES, the PAs of the same beam position are nearly the same, while those of different beam positions, even adjacent beam positions, may be rather different. Consequently, ES can be distinguished from MS based on this difference.

The flowchart of the proposed method in this paper is shown in Figure 2. Firstly, some processing (normalization and ASP estimation) is carried out for beam sequence extraction. Then, 1D ES is recognized based on the features 


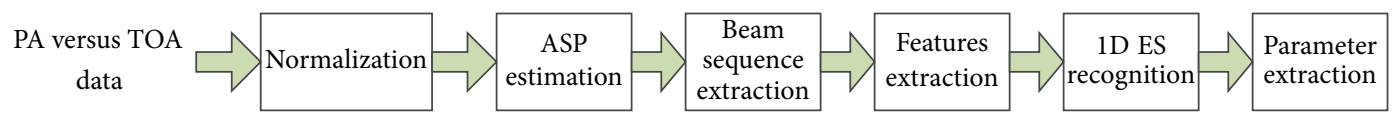

FIgURE 2: The flowchart of the methodology.

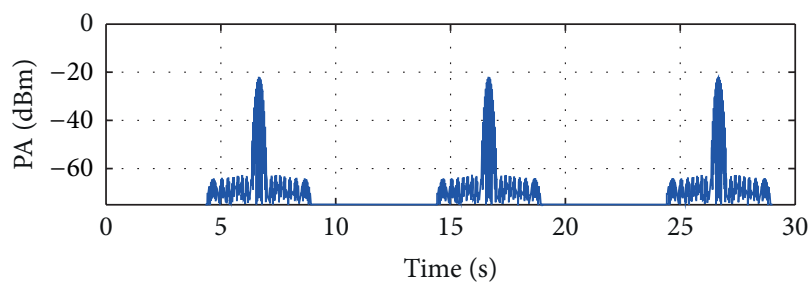

(a)

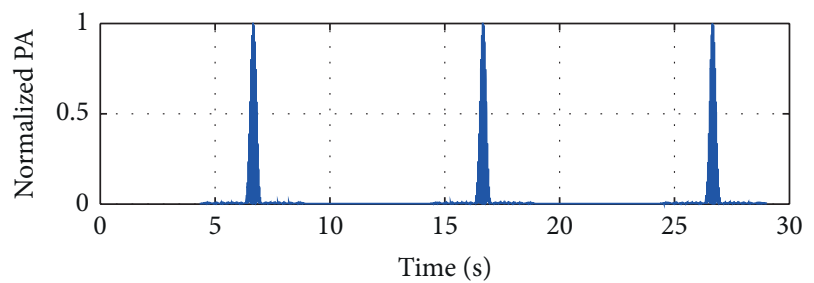

(b)

Figure 3: An example PA versus a TOA signal for 3D radar and its normalized sequence.

extracted from the beam sequence by utilizing the RC [16]. Finally, two vital parameters for 1D ES are extracted, namely, beam position number in elevation and pulse number in a beam position.

3.1. Normalization. Assume that the received or simulated PA and TOA data are $a[m]$ and $t[m]$, respectively, $m=$ $0,1, \ldots, M-1$, where $M$ is the pulse number. The input signal should have at least two cycles of the antenna scan for ASP estimation. Figure 3(a) shows an example PA versus a TOA signal with three periods from a 3D radar of Hamming weighting type. In order to eliminate the influence of propagation, normalization is needed for PA data. While PA data are in $\mathrm{dBW}$ (decibel relative to Watt) scale, the maximum value of which may be zero, the data is transformed from dBW to Volt (V) first and normalized subsequently. Consider

$$
\begin{gathered}
a_{V}[m]=10^{a[m] / 20}, \quad m=0,1, \ldots, M-1, \\
\widetilde{a}[m]=\frac{a_{V}[m]}{\max \left(\left\{a_{V}\right\}\right)}, \quad m=0,1, \ldots, M-1,
\end{gathered}
$$

where $\max (\cdot)$ stands for the maximum value of the sequence in round brackets. In this paper, a sequence can also be denoted by a letter or letters in curly brackets, for example, $\left\{a_{V}\right\}$.

As a result of normalization, the example sequence in Figure 3(a) is transformed to the sequence shown in Figure 3(b).

3.2. ASP Estimation. The AST analysis is based on PA sequence with only one period, so we estimate the ASP first. The ASP estimation algorithm used in literature [13] is applied in this paper.

In modern EW, various types of PRI modulation are employed, such as constant, jittered, staggered, sliding, wobulated, and D \& S (Dwell and Switch) [5]. It will make the sampling of PA data nonuniform and result in complex succeeding processing. In order to estimate the ASP, it is better to resample the received data uniformly.
Resampling with the shortest pulse interval is a simple measure, while it does not take into account the PRI modulation. For exactness, we determine the sampling interval after the analysis of PRI modulation. Different PRI analysis methods such as CDIF [17], SDIF [18], PRI transform [19], and plan transform [20] can be used here and different resampling intervals are chosen for different PRI modulation. For example, the signal with stagger-type PRI is resampled with the highest PRI in the stagger levels [13] and that with jitter-type PRI is resampled with the average PRI. It is well known that PRI analysis is crucial to radar signal sorting. Consequently, resampling signals based on PRI modulation information can maintain as many original signals as possible and reduce the influence of dropped and false pulses. Furthermore, the result of PRI analysis can also be useful in the next section. During the resampling process, interpolation is performed if the signal value is not available at a particular instant. Different interpolation techniques can be used, including nearest point, linear, polynomial, Gaussian, sinc, or spline interpolation. For simplicity, we choose nearest point interpolation here, and the points where no pulses are detected by receiver are filled with zeros.

Suppose that the resampling interval chosen based on the result of PRI analysis is $T_{p}$ (for the example signal in Figure $3 T_{p}$ is chosen to $0.002 \mathrm{~s}$ ), and the resampled sequence is $x[n], n=0,1, \ldots, N-1$, where $N$ is the sequence length.

The normalized autocorrelation coefficients of the sequence $\{x\}$ are calculated to estimate the ASP:

$$
\begin{array}{r}
r_{x x}[l]=\frac{\sum_{n=0}^{W-1} x[n] x[n+l]}{\sqrt{\sum_{n=0}^{W-1} x^{2}[n]} \sqrt{\sum_{n=0}^{W-1} x^{2}[n+l]}}, \\
l=0,1, \ldots, N-W,
\end{array}
$$

where $l$ is the lag variable and $W$ is the window length that is chosen to compromise between computational complexity and accuracy. Since the sequence $\{x\}$ is assumed to have at least two periods, $W$ is chosen as $N / 2$ in this paper.

The first maximum value of the sequence $r_{x x}[l]$ is found and the corresponding lag value $l_{d}$ is assigned as the period. 


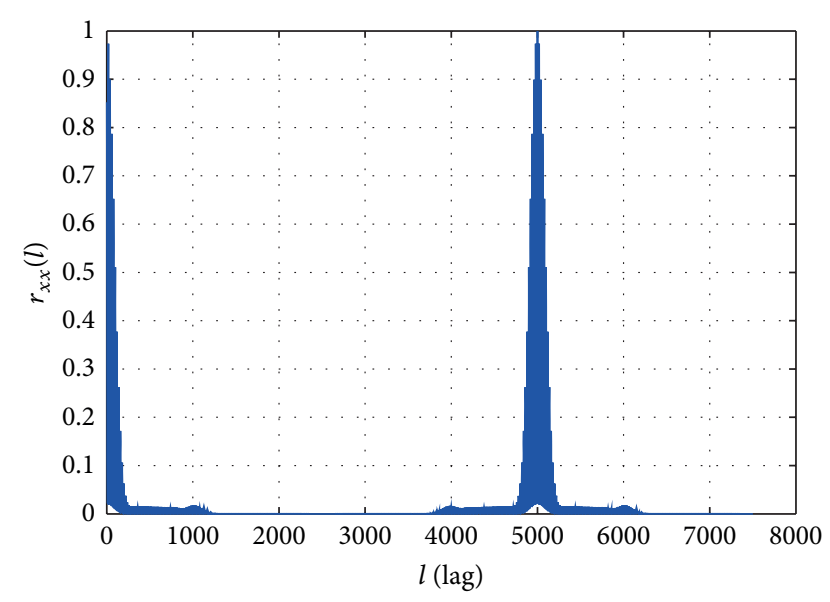

FIGURE 4: Normalized autocorrelation coefficients of example sequence.

Hence the ASP can be obtained by $T_{A}=l_{d} \cdot T_{p}$. The normalized autocorrelation coefficients of the example sequence are illustrated in Figure 4 , from which we can obtain $l_{d}=5000$, so that $T_{A}=10 \mathrm{~s}$.

3.3. Beam Sequence Extraction. After ASP estimation, the signal of one scan circle can be extracted for following processing. During the extraction, make sure the circles are integrated and the main beam is located in the middle of every circle. Assume that the sequence with one period extracted from the normalized input signal before resampling is $\left\{\widetilde{a}\left[n_{1}\right], \ldots, \widetilde{a}\left[n_{2}\right]\right\}$.

When the radar beam steers through the location of the EW receiver in a scanning circle, the received PA reaches the maximum in the period. Therefore, we can locate the maximum main beam by finding the maximum PA first. In literature [13], the main beam sequence is extracted by using the two index points on each side of the maximum point where the PA drops to $0.01 \mathrm{mV}$. However, it does not take into account the ES. Unlike the analysis of MS which is based on only main beams, that of ES is based on beam group, including both main beam and adjacent side beams. Consequently, the key of beam sequence extraction is to find the edge of side beams.

Because of the quick switch of beam direction to ES, the change of received PA data may be drastic. The PA may drop to $0.01 \mathrm{mV}$ or other PA threshold values even during the main beam scanning; hence, the method of beam extraction based on PA threshold is no longer valid. In this study, we extract the beam sequence based on received TOA data.

The PAs of side beams are commonly much less than those of main beam. When the PA is below the sensitivity level of the EW receiver, the pulse will not be intercepted. Most signals of side beams, except the first side beam, may be undetected by EW receiver. As a result, there will be some intervals between detected pulses much longer than ordinary PRIs.

In order to determine the edges of side beams, we set a PRI threshold $H_{t}$ according to the PRI analysis result in
Section 3.2 and then find two nearest PRIs that are longer than $H_{t}$ on both sides of the main beam. The concrete procedures of beam sequence extraction are given below.

Step 1: Find the maximum PA $\widetilde{a}\left[M_{a}\right], n_{1}<M_{a}<n_{2}$. This step is used to locate the main beam in the scan circle.

Step 2: Initialization. Set bit $=1, i=0$.

Step 3: Compute the PRI on the right side of the main beam one by one. Let $i=i+1 ; m=M_{a}-i \times$ bit; compute $t^{\prime}=t[m+1]-t[m]$.

Step 4: If $t^{\prime}<H_{t}, H_{t}$ being the threshold of pulse interval, repeat Step 3; otherwise if $t^{\prime} \geq H_{t}$, the edge of right side beam can be determined; denote current $m$ by $m_{1}$.

Step 5: Initialization. Set bit $=-1, i=0$.

Step 6: Compute the PRI on the left side of the main beam one by one. Let $i=i+1 ; m=M_{a}-i \times$ bit; compute $t^{\prime}=t[m]-t[m-1]$.

Step 7: If $t^{\prime}<H_{t}$, repeat Step 6; otherwise if $t^{\prime} \geq H_{t}$, the edge of left side beam can be determined; denote current $m$ by $m_{2}$.

Step 8: Extract the beam sequence $\left\{a_{m}\right\}=\left\{\widetilde{a}\left[m_{1}\right], \widetilde{a}\left[m_{1}+\right.\right.$ $\left.1], \ldots, \widetilde{a}\left[m_{2}-1\right], \widetilde{a}\left[m_{2}\right]\right\}$ and the corresponding TOA sequence $\left\{t\left[m_{1}\right], t\left[m_{1}+1\right], \ldots, t\left[m_{2}-1\right], t\left[m_{2}\right]\right\}$.

Besides the first side beams, some other side beams may also be extracted after these steps. It will not affect the following recognition. The beam sequence extracted from the example sequence in Figure 3 is depicted in Figure 5(a), and Figure 5(b) shows a typical beam sequence of MS extracted by the above procedure.

3.4. Feature Extraction. According to the previous analysis, the crucial difference of PA sequence between MS and ES lies at the points where beams switch. Since the antenna beam moves inertially for MS, the measured PAs change continuously. For ES, the PAs of the same beam position show slight variation, while when beam direction changes, there may be a dramatic change of PAs. Therefore, it is necessary to analyze the variations of PA sequence, and the processing that first occurs is calculating the difference sequence.

At first, the first-order difference sequence of the PA data is calculated:

$$
d_{1}[m]=a_{m}[m+1]-a_{m}[m], \quad m=0,1, \ldots, N_{d},
$$

where $N_{d}=m_{2}-m_{1}$. The first-order difference sequences of example sequences in Figure 5 are illustrated in Figure 6. It is easy to see that the sequence $\left\{d_{1}\right\}$ reflects the amplitude variation of adjacent pulses. For ES, the values of most points in sequence $\left\{d_{1}\right\}$ are close to 0 , while at the points where main or side beams switch, the values reach local maxima or minima. The maximum occurs at the point where the main beam changes to the first side beam, and its value is slightly less than 1. Similarly for MS, there are also local maxima or minima where main or side beams switch, and the maximum also occurs at the switch point between the main beam and 


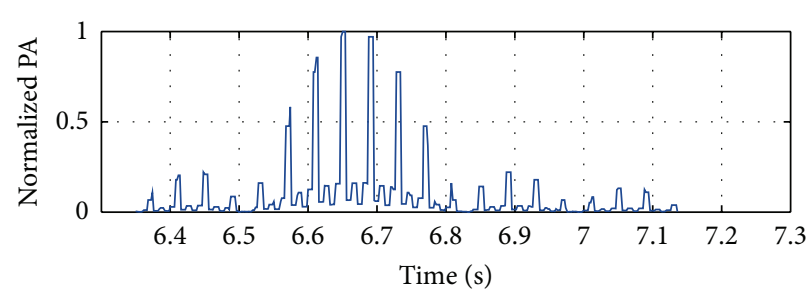

(a) ES

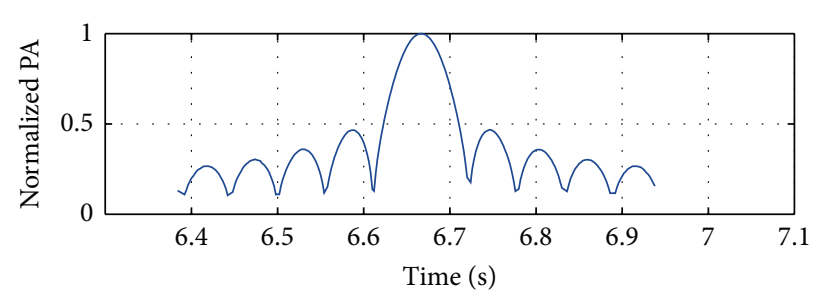

(b) MS

Figure 5: Beam sequence.

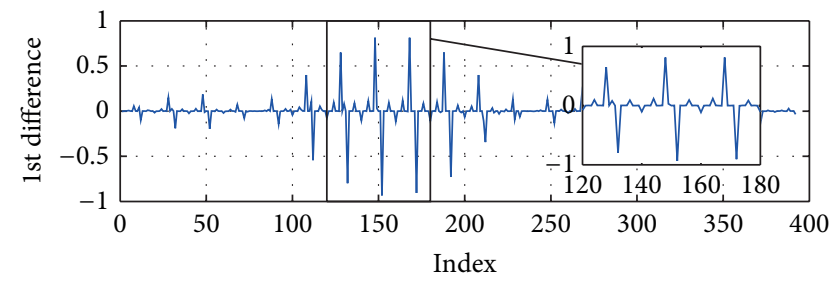

(a) ES

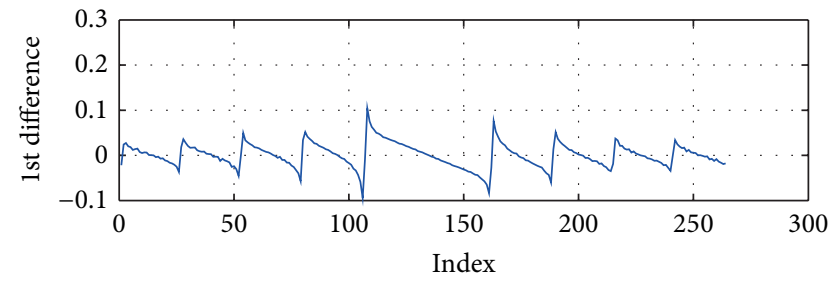

(b) MS

FIGURE 6: First-order difference sequence.

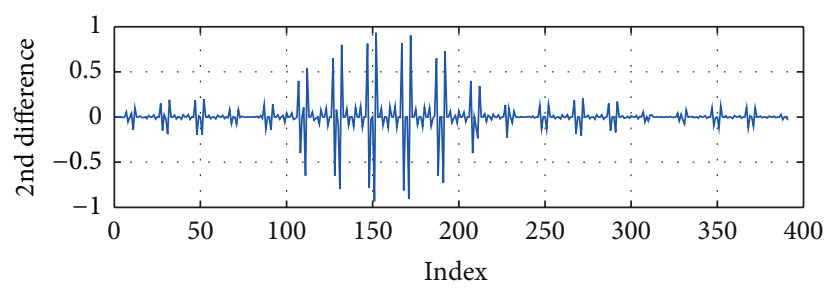

(a) ES

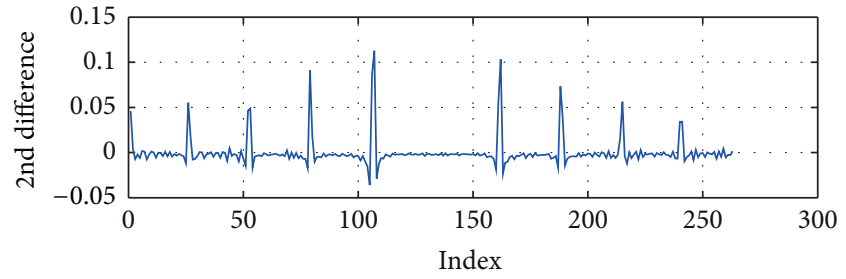

(b) MS

FIGURE 7: Second-order difference sequence.

side beam. What is different is that the sequence $\left\{d_{1}\right\}$ shows gradual change at most points for MS, and the maximum value is much less than 1 .

Because most values of sequence $\left\{d_{1}\right\}$ for ES are near 0 , we further calculate the second-order difference sequence:

$$
d_{2}[m]=d_{1}[m+1]-d_{1}[m], \quad m=0,1, \ldots, N_{d}-1
$$

Then there may be slight variation between $d_{2}[m]$ and $d_{1}[m+$ $1]$ for most $m$ for ES. The second-order difference sequences of example sequences in Figure 5 are illustrated in Figure 7.

In order to accomplish the recognition, we have attempted to study a number of different features to identify ES, but the best results were obtained with the feature described below.

By comparing the first-order difference sequences in Figure 6 with the second-order ones in Figure 7, we found that there are some notable differences between ES and MS. For ES, the first- and second-order difference sequences seem similar at most points, except the points where main or side beams change while for MS, the two sequences are quite different. Therefore, we select RC as the feature to distinguish MS and ES.

Definition 1. Suppose that discrete signal sequences $\left\{S_{1}(i), i=\right.$ $0,1, \ldots, N\}$ and $\left\{S_{2}(j), j=0,1, \ldots, N\right\}$ are one-dimensional and nonnegative; that is, $S_{1}(i) \geq 0 ; S_{2}(i) \geq 0 ; i=0,1, \ldots, N$. $\mathrm{RC}$ of $\left\{S_{1}\right\}$ and $\left\{S_{2}\right\}$ is defined as

$$
C_{r}=\frac{\sum S_{1}(i) S_{2}(i)}{\left(\sqrt{\sum S_{1}^{2}(i)} \cdot \sqrt{\sum S_{2}^{2}(i)}\right)},
$$

where all points of signal sequences $\left\{S_{1}(i)\right\}$ and $\left\{S_{2}(j)\right\}$ are not 0 . Obviously, we can get $0 \leq C_{r} \leq 1$.

In order to obtain the RC from the two difference sequences, we set $\left\{S_{2}(i)=\left|d_{2}(i)\right|, i=0,1, \ldots, N_{d}-1\right\}$, and $\left\{S_{1}\right\}$ is given by the following formula:

$$
S_{1}(i)=\max \left(\left|d_{1}(i+1)\right|,\left|d_{2}(i)\right|\right), \quad i=0,1, \ldots, N_{d}-1 .
$$

Then the RC can be calculated using formula (7). The value of $C_{r}$ is large for ES and nearly equal to 1, while it is much less for MS. 


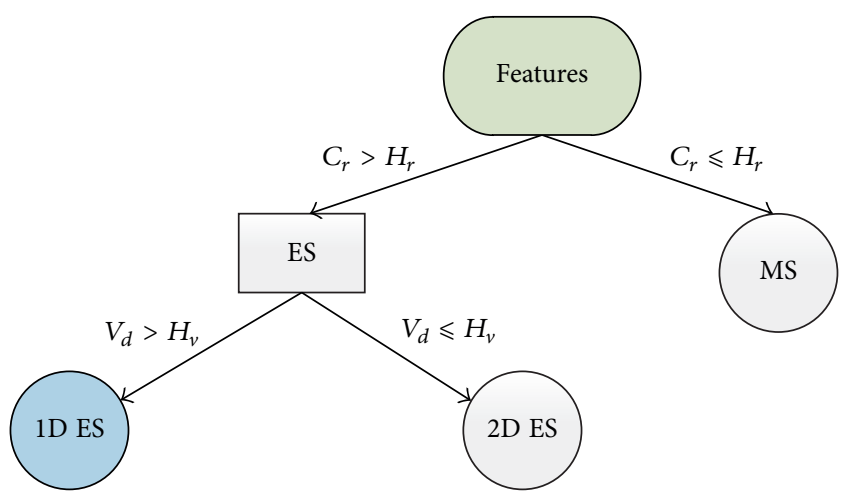

FIGURE 8: The DT structure for recognition of 1D ES.

Furthermore, in order to differentiate 1D ES from 2D ES, we further process the first-order difference sequence $\left\{d_{1}\right\}$. First, pick up the elements less than $H_{a}$ in sequence $\left\{\left|d_{1}\right|\right\}$; $H_{a}$ is the threshold. Hence, the picked points present the variations among a beam position for ES. Then, compute the variance of picked elements, which is denoted by $V_{d}$. Because there is no beam movement among a beam position for $2 \mathrm{D}$ $\mathrm{ES}, V_{d}$ of $2 \mathrm{D}$ ES is commonly less than that for $1 \mathrm{D} \mathrm{ES}$, for which beam still moves inertially in azimuth.

3.5. Recognition. The recognition of $1 \mathrm{D}$ ES has two stages: first distinguishing ES from MS and then distinguishing 1D ES from 2D ES.

After extracting the two features, we can accomplish the recognition by utilizing a classifier, such as naive Bayes, decision tree (DT) [21], clustering analysis, artificial neural networks [22], and support vector machine [22]. Based on the recognition procedure in this paper, a DT classifier based on clustering analysis is chosen to recognize $1 \mathrm{D} \mathrm{ES}$.

A DT classifier is one of the most intuitive and natural classifiers and it is fast, comprehensible, and easy to visualize. A DT consists of two kinds of nodes: an internal node represents a decision rule and a leaf node shows the result of a decision. The DT structure used for recognition of $1 \mathrm{D}$ ES is shown in Figure 8.

Clustering analysis is the task of grouping a set of objects in such a way that objects in the same cluster are more similar to each other than to those in other clusters. It is used in many fields, including pattern recognition, machine learning, and information retrieval. In this paper, clustering is mainly used to determine the threshold of decision rule in DT. The Euclidean distance criterion is most commonly employed in clustering, and the Euclidean distance between $z_{1}=\left(z_{11}, \ldots, z_{1 j}\right)$ and $z_{2}=\left(z_{21}, \ldots, z_{2 j}\right)$ of instances is defined as

$$
d\left(z_{1}, z_{2}\right)=\sqrt{\sum_{i=1}^{j}\left(z_{1 i}-z_{2 i}\right)^{2}}
$$

Since there is only one feature applied in each decision rule, the cluster center can be easily obtained by taking the mean of train data with every AST. For simplicity, we

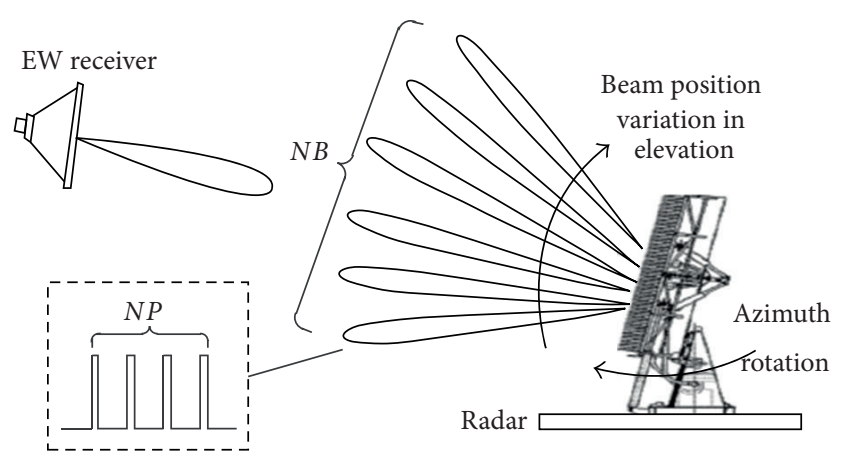

Figure 9: A sketch map of $N B$ and $N P$ for 3D radar with $1 D$ ES.

select the median of the cluster centers of two leaf nodes as the threshold in each decision. Then $H_{r}$ and $H_{v}$ can be determined, and recognition can be carried out according to the DT in Figure 8.

3.6. Parameter Extraction. This paper mainly considers the 1D ES of 3D radar, which employs a judicious mix of electronic beam steering orderly in elevation and mechanically movement in azimuth. After identification of the 1D ES from MS and 2D ES, we need to extract some crucial parameters of $1 \mathrm{D}$ ES of 3D radar. Besides ASP, the key parameters of $1 \mathrm{D}$ electronic scanning are the beam position number in elevation (defined by $N B$ ) and pulse number in a beam position (defined by $N P$ ), which are important to estimate the beam direction of $3 \mathrm{D}$ radar, as shown in Figure 9. For simplicity, both $N B$ and NP are assumed to be constant in this study. The $N B$ and NP are 6 and 4, respectively, in Figure 9.

The parameter extraction of $1 \mathrm{D}$ ES of $3 \mathrm{D}$ radar is mainly based on the beam sequence $\left\{a_{m}\right\}$ extracted in Section 3.3. From the first-order difference sequence of the sequence $\left\{a_{m}\right\}$ shown in the subgraph of Figure 6(a), we can see that there exist a peak and a trough at two sides of every beam position, which result from the beam position variation in elevation. Figure 10 depicts the first difference sequence of the subgraph and its corresponding PA sequence. Consequently, we can extract $N P$ by determining the interval between the highest peak and the lowest trough of the beam position with maximum PA. Firstly, locate the maximum PA of sequence $\left\{a_{m}\right\}$, denoted by $a_{m}\left[M_{b}\right], 0<M_{a}<N_{d}$. Obviously, $a_{m}\left[M_{b}\right]$ is equal to $\widetilde{a}\left[M_{a}\right]$, which is extracted in Section 3.3. Then, as for the sequence $\left\{d_{1}\right\}$, locate the nearby highest peak before $d_{1}\left[M_{b}\right]$ (denoted by $d_{1}\left[M_{p}\right]$ ) and the nearby lowest trough after $d_{1}\left[M_{b}\right]$ (denoted by $d_{1}\left[M_{t}\right]$ ). For the example sequence shown in Figure $5(\mathrm{~b})$, both $d_{1}\left[M_{p}\right]$ and $d_{1}\left[M_{t}\right]$ are marked with red circles in Figure 10(b). Hence, the pulse number in a beam position can be calculated by $N P=M_{t}-M_{p}$.

In order to extract $N B$, the steer circle in elevation of $1 \mathrm{D} E S$, that is, the pulse number in an elevation circle, should be determined. An example of an elevation circle sequence is marked with red in Figure 10(a). Because the beam sequences of two adjacent elevation circles are similar in amplitude, we can compare the entire sequence $\left\{a_{m}\right\}$ with reference sequence to estimate the elevation circle interval, and normalized cross-correlation coefficients are applied 


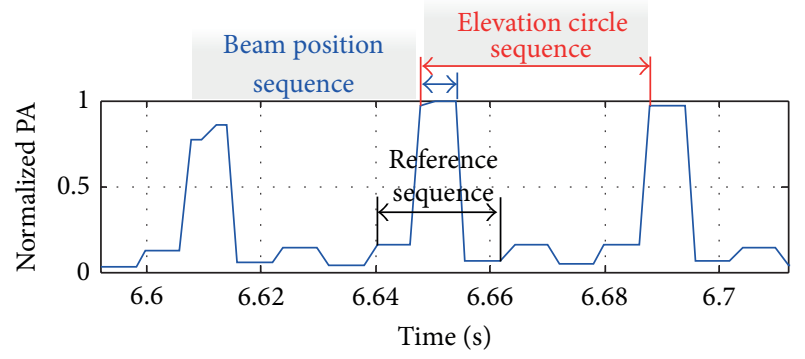

(a) Normalized PA

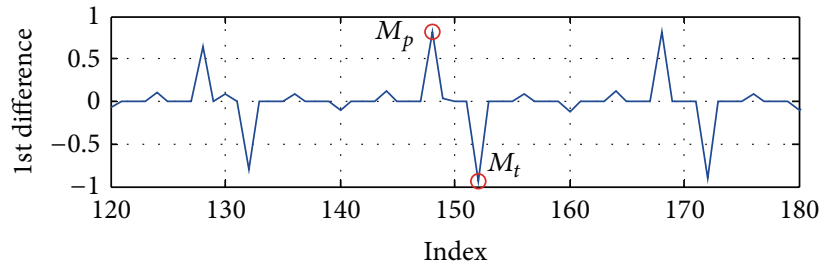

(b) 1st difference

FIGURE 10: An extracted PA sequence and first difference sequence for 1D ES.

here. Firstly, we determine the reference sequence. After NP extraction, the beam position sequence with maximum PA can be obtained easily, namely, $\left\{a_{m}\left[M_{p}+1\right], \ldots, a_{m}\left[M_{t}\right]\right\}$, marked with blue in Figure 10(a). Then we select the PA data of the maximum beam position and the adjacent beam positions at its both sides as the reference sequence $\{y[m], m=$ $\left.0,1, \ldots, N_{y}-1\right\}$, marked with black in Figure 10(a). Hence, $\{y\}=\left\{a_{m}\left[M_{p}+1-N P\right], \ldots, a_{m}\left[M_{t}+N P\right]\right\}$, and $N_{y}=3 \times N P$. Calculate the mean square errors (MSE) of sequence $\{y\}$ and $\left\{a_{m}\right\}$ :

$$
\begin{array}{r}
e_{a y}[l]=\sqrt{\sum_{n=0}^{N_{y}-1}\left(y[n]-a_{m}[n+l]\right)^{2}}, \\
l=0,1, \ldots, N_{d}+1-N_{y} .
\end{array}
$$

The MSE sequence of the example sequence is shown in Figure 11, from which we can see that in sequence $\left\{e_{a y}\right\}$ there are several troughs, which are located where there are main beam positions. Among these troughs, the lowest one whose value is equal to 0 represents the maximum main beam position, namely, the reference sequence $\{y\}$. Furthermore, the secondary lowest trough represents the adjacent main beam position. Let $N_{m}$ denote the interval of the two troughs which are marked with red circles in Figure 11 so that $N B$ can be obtained by $N B=N_{m} / N P$.

\section{Simulations}

In order to illustrate the proposed algorithm for recognition of $1 \mathrm{D}$ ES, several simulations are discussed in this section.

4.1. Recognition. At first, 60 data sequences are generated for training of feature extraction and recognition described in Sections 3.4 and 3.5: 20 from 1D ES, 20 from 2D ES, and the remaining from MS. All the ES data are generated by the simulator designed in Section 2 and the MS data by the antenna scan pattern simulator in literature [23]. While generating the simulated data, we have varied some different parameters (e.g., period, weightings, angles, and so on) so that the simulated data resemble the actual data from different realistic scenarios as much as possible.

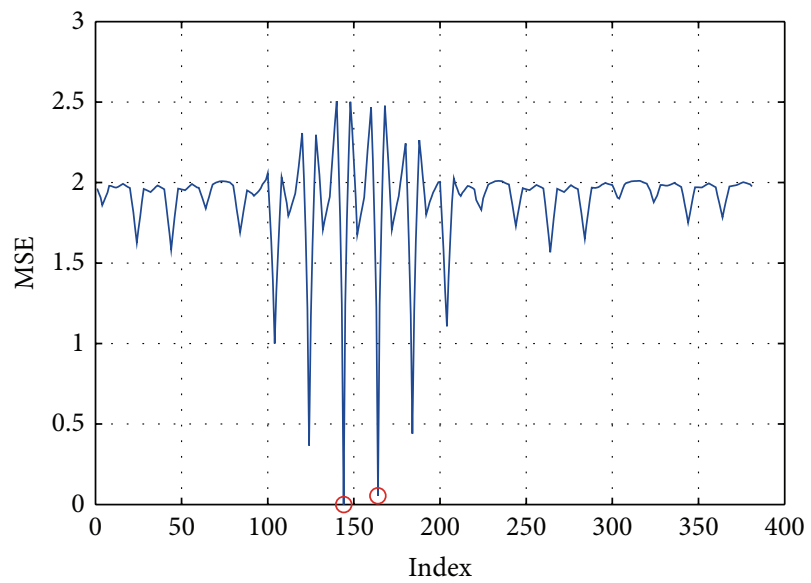

FIgURE 11: The MSE sequence.

TABLE 1: Recognition results.

\begin{tabular}{lcccc}
\hline Input & \multicolumn{3}{c}{ Classification } & Recognition rate (\%) \\
& 1D ES & 2D ES & MS & 88 \\
\hline 1D ES & $\mathbf{8 8}$ & 11 & 1 & 92 \\
2D ES & 8 & $\mathbf{9 2}$ & 0 & 95 \\
MS & 4 & 1 & $\mathbf{9 5}$ & \\
\hline
\end{tabular}

The features extracted from the simulated data sequence are depicted in Figure 12. We can see that the feature $C_{r}$ is distinguishable. Most $C_{r}$ values are equal to 1 for both 1D ES and $2 \mathrm{D}$ ES. Although the values of $C_{r}$ are above 0.7 for MS, they are still less than those for ES. The threshold $H_{r}$ is set to 0.88 based on the train data. Moreover, the $V_{d}$ value for $1 \mathrm{D}$ ES is generally greater than that for 2D ES, and the threshold $H_{v}$ is set to 0.00031 .

The number of simulated samples is increased to 100 for each AST for recognition. Table 1 presents the specific recognition results. The data illustrate that the method proposed in this paper is effective to distinguish ES from MS. The recognition rates of different ASTs are equal to or above $88 \%$ and the classification accuracy for ES (1D and 2D) even reaches $99.5 \%$. It is suggested to refer to literature [13] for further classification of specific MS type, such as circular scan, sector scan, raster scan, and helical scan. 


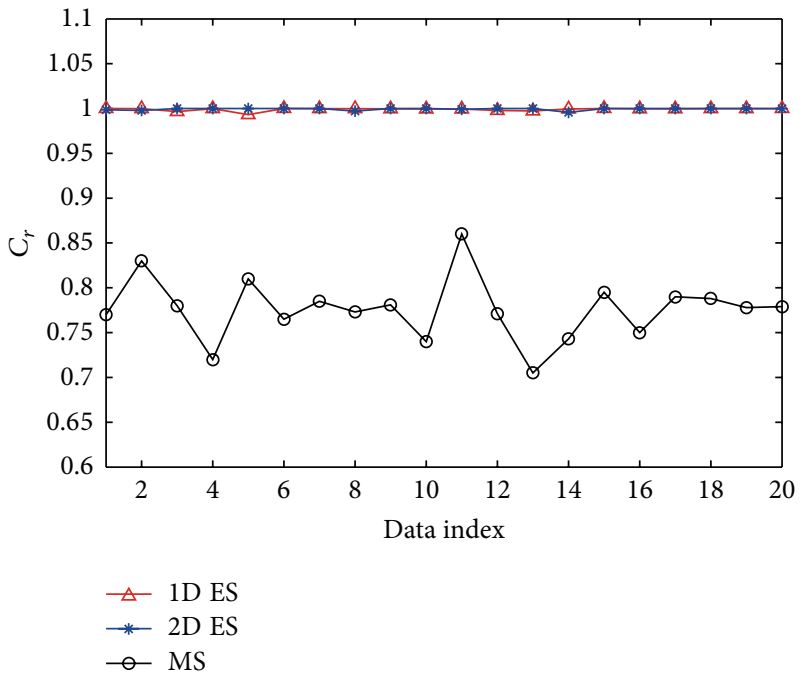

(a) $C_{r}$

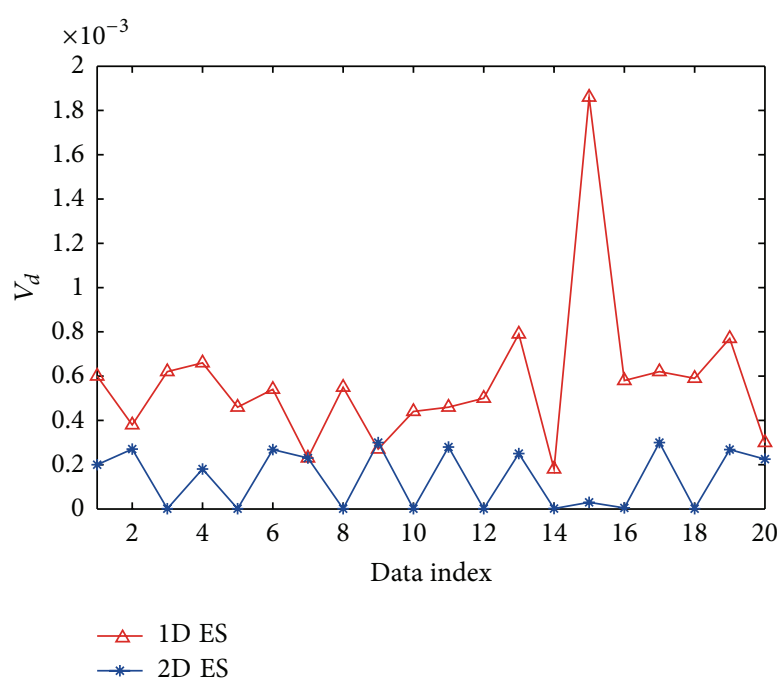

(b) $V_{d}$

Figure 12: The extracted features.

TABLE 2: Simulation scenarios.

\begin{tabular}{lcccc}
\hline Scenario & MS $^{*}$ & Scope In ?** $^{*}$ & NP extraction rate (\%) & NB extraction rate (\%) \\
\hline 1 & Circular & Yes & 95 & 95 \\
2 & Sector & Yes & 94 & 94 \\
3 & Circular & No & 94 & 93 \\
4 & Sector & No & 89 & 89 \\
\hline
\end{tabular}

The mechanical scanning type applied by $3 \mathrm{D}$ radar in azimuth.

** Does the EW receiver locate in the elevation scope of $3 \mathrm{D}$ radar?

4.2. Parameter Extraction. In order to validate the parameter extraction for 1D ES, 4 different scenarios are considered in this simulation, which are listed in Table 2. When the EW receiver does not locate in the elevation scope of $3 \mathrm{D}$ radar, it can only receive the signals from the side beams of 3D radar. The simulated samples in which the acquired pulses are not sufficient for parameter extraction are aborted.

Unlike estimated parameters (e.g., ASP) which are available in a tolerance range, the main parameters extracted in this paper ( $N P$ and $N B$ ) will be invalid if the extracted values are incorrect. Therefore, the extraction accuracy is used to evaluate the performance of parameter extraction here. The extraction result is illustrated in Table 2. It can be observed that the correct extraction rate of $N P$ is almost equal to that of $N B$. The reason is that the extraction of $N B$ is based on the result of $N P$ extraction. The extraction accuracies are over $89 \%$ and with slight difference for all 4 scenarios. It can be deduced that the parameter extraction is effective even by the side beams signals.

\section{Conclusions}

In this paper, we studied the problem of recognition and parameter extraction of $1 \mathrm{D}$ ES for $3 \mathrm{D}$ radar on which no clear and accessible study is available in the open literature. The main contribution of this work is making a maiden attempt to resolve the problem and providing an effective method. Firstly, an ES pattern simulator to synthesize PA data is designed. Then, the methodology for recognition and parameter extraction of $1 \mathrm{D}$ ES for $3 \mathrm{D}$ radar is explained in detail. Finally, the algorithm is validated by simulation.

Our study will be beneficial to $3 \mathrm{D}$ radar identification for ELINT and ESM systems. Moreover, it is also useful for estimating the beam direction of $3 \mathrm{D}$ radar. Our main following work is to validate the algorithm by real radar data acquired by EW receivers. To achieve the purpose, some experiments are going to be designed in the future.

\section{Conflict of Interests}

The authors declare that there is no conflict of interests regarding the publication of this paper.

\section{Acknowledgment}

This work is supported by the National Natural Science Foundation of China (no. 61201336).

\section{References}

[1] J. Wisniewski and M. Mazur, "Naval 3D radar in C band," in Proceedings of the 14th IEEE International Conference on Microwaves, Radar and Wireless Communications (MIKON '02), vol. 3, pp. 843-846, 2002. 
[2] F. Orsini, B. di Loreto, and A. Giacomini, "3D X-band tactical acquisition radar (X-TAR 3D)," in Proceedings of the European Radar Conference, pp. 589-592, October 2009.

[3] D. L. Adamy, EW 102: A Second Course in Electronic Warfare, Artech House, Boston, Mass, USA, 2004.

[4] A. Irci, A. Saranli, and B. Baykal, "Study on Q-RAM and feasible directions based methods for resource management in phased array radar systems," IEEE Transactions on Aerospace and Electronic Systems, vol. 46, no. 4, pp. 1848-1864, 2010.

[5] R. G. Wiley, ELINT: The Interception and Analysis of Radar Signals, Artech House, Boston, Mass, USA, 2006.

[6] M. Zhu, K.-C. Fu, X.-Y. Huang, S.-D. Wu, and W.-D. Jin, "A novel recognition approach for radar emitter signals based on on-line independent support vector machines," Advances in Computer Science and Its Applications, vol. 2, no. 3, pp. 390-396, 2013.

[7] D. H. A. Maithripala and S. Jayasuriya, "Radar deception through phantom track generation," in Proceedings of the American Control Conference (ACC '05), pp. 4102-4106, June 2005.

[8] B. Falk, “Antenna Pattern Generator," US Patent 3,540,046, 1970.

[9] A. B. Evan Jr., "Digital antenna pattern generator for radar simulation," US Patent 4,008,476, 1977.

[10] T. E. Zaczek, “Antenna scan pattern generator," US Patent 4327417, 1982.

[11] Y.-H. Kim, W.-J. Kim, K.-H. Song, J.-W. Han, and H.-N. Kim, "Modeling of a radar signal for scan pattern," in Proceedings of the IEEE Military Communications Conference (MILCOM '09), pp. 1-6, Vancouver, Canada, October 2009.

[12] T. H. Greer, "Automatic recognition of radar scan type," US Patent 6,697,007, 2004.

[13] B. Barshan and B. Eravci, "Automatic radar antenna scan type recognition in electronic warfare," IEEE Transactions on Aerospace and Electronic Systems, vol. 48, no. 4, pp. 2908-2931, 2012.

[14] X.-S. Wang, S.-P. Xiao, D.-J. Feng, and F. Zhao, Modeling and Simulation of Modern Radar and Electronic Warfare Systems, Publishing House of Electronics Industry, Xian, China, 2010.

[15] B. R. Mahafza and A. Z. Elsherbeni, MATLAB Simulations for Radar Systems Design, CRC Press, Boca Raton, Fla, USA, 2004.

[16] G.-X. Zhang and X. Li, "A new recognition system for radar emitter signals," Kybernetes, vol. 41, no. 9, pp. 1351-1360, 2012.

[17] H. K. Mardia, "New techniques for the deinterleaving of repetitive sequences," IEE proceedings, vol. 136, no. 4, pp. 149$154,1989$.

[18] D. J. Milojevic and B. M. Popovic, "Improved algorithm for the deinterleaving of radar pulses," IEE Proceedings F, vol. 139, no. 1, pp. 98-104, 1992.

[19] D. J. Nelson, "Special purpose correlation functions for improved signal detection and parameter estimation," in Proceedings of the International Conference on Acoustics, Speech, and Signal Processing (ICASSP '93), pp. 73-76, 1993.

[20] P. Wei, L. Qiu, and $\mathrm{H}$. Wu, "The sorting of radar pulse signal based on plane transformation," in Proceedings of the International Conference on Systems and Informatics (ICSAI '12), pp. 326-328, May 2012.

[21] W.-T. Lo, Y.-S. Chang, R.-K. Sheu, C.-C. Chiu, and S.-M. Yuan, "CUDT: a CUDA based decision tree algorithm," The Scientific World Journal, vol. 2014, Article ID 745640, 12 pages, 2014.
[22] Q.-D. Wu, B. Yan, C. Zhang, L. Wang, G.-B. Ning, and B. $\mathrm{Yu}$, "Displacement prediction of tunnel surrounding rock: a comparison of support vector machine and artificial neural network," Mathematical Problems in Engineering, vol. 2014, Article ID 351496, 6 pages, 2014.

[23] B. Eravcg, Automatic radar antenna scan analysis in electronic warfare [M.S. thesis], Department of Electrical and Electronics Engineering, Bilkent University, Ankara, Turkey, 2010. 

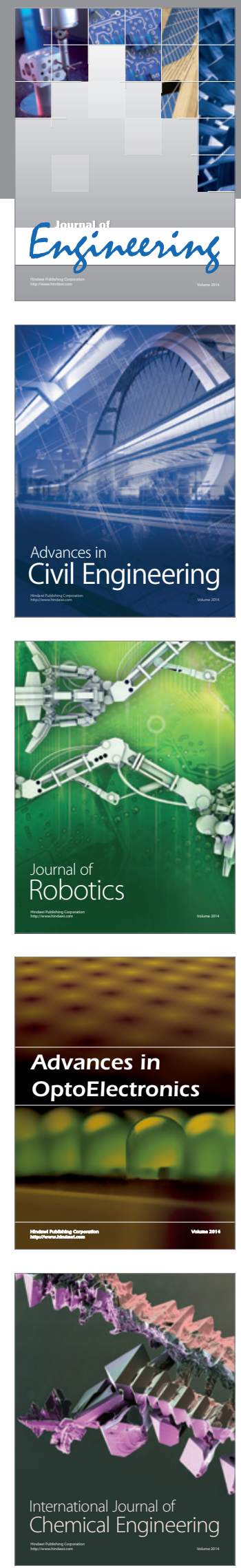

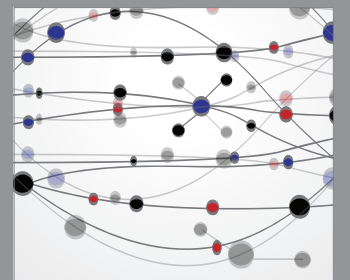

The Scientific World Journal
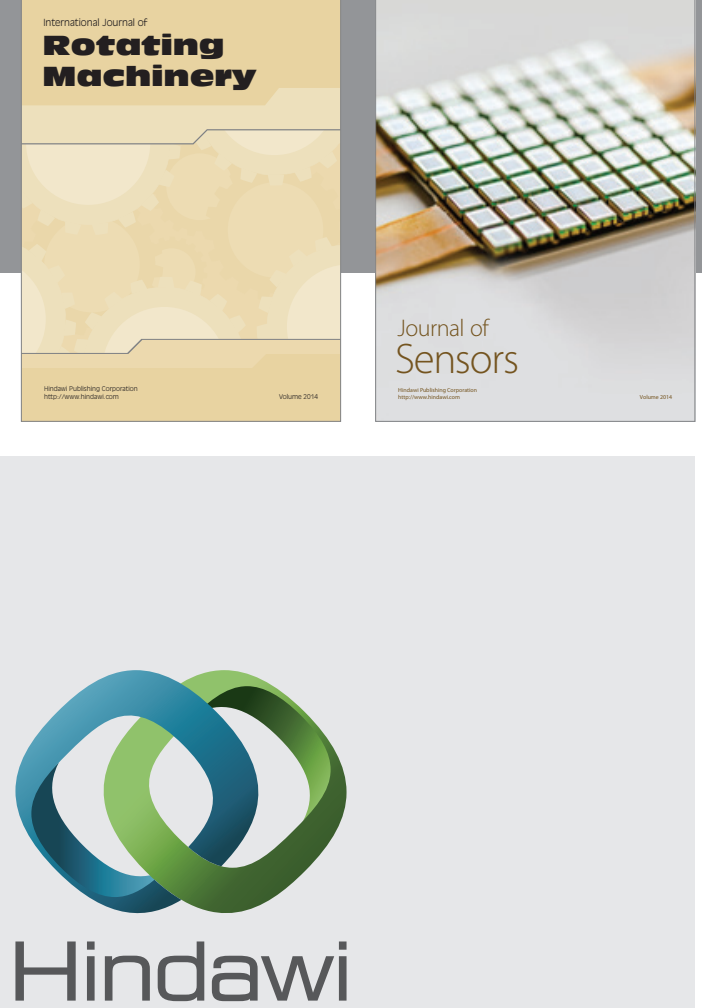

Submit your manuscripts at http://www.hindawi.com
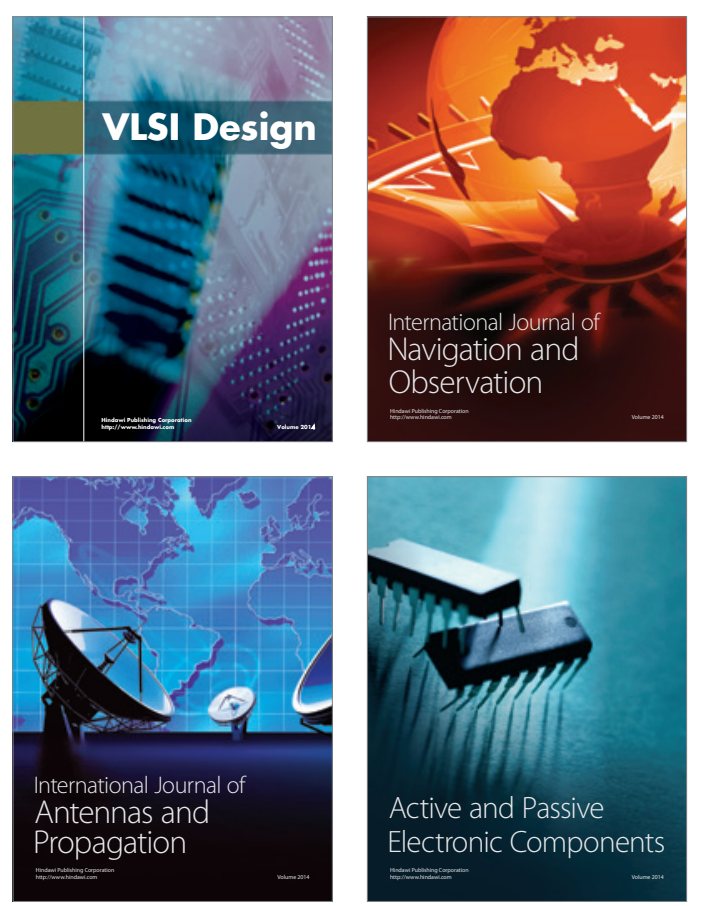
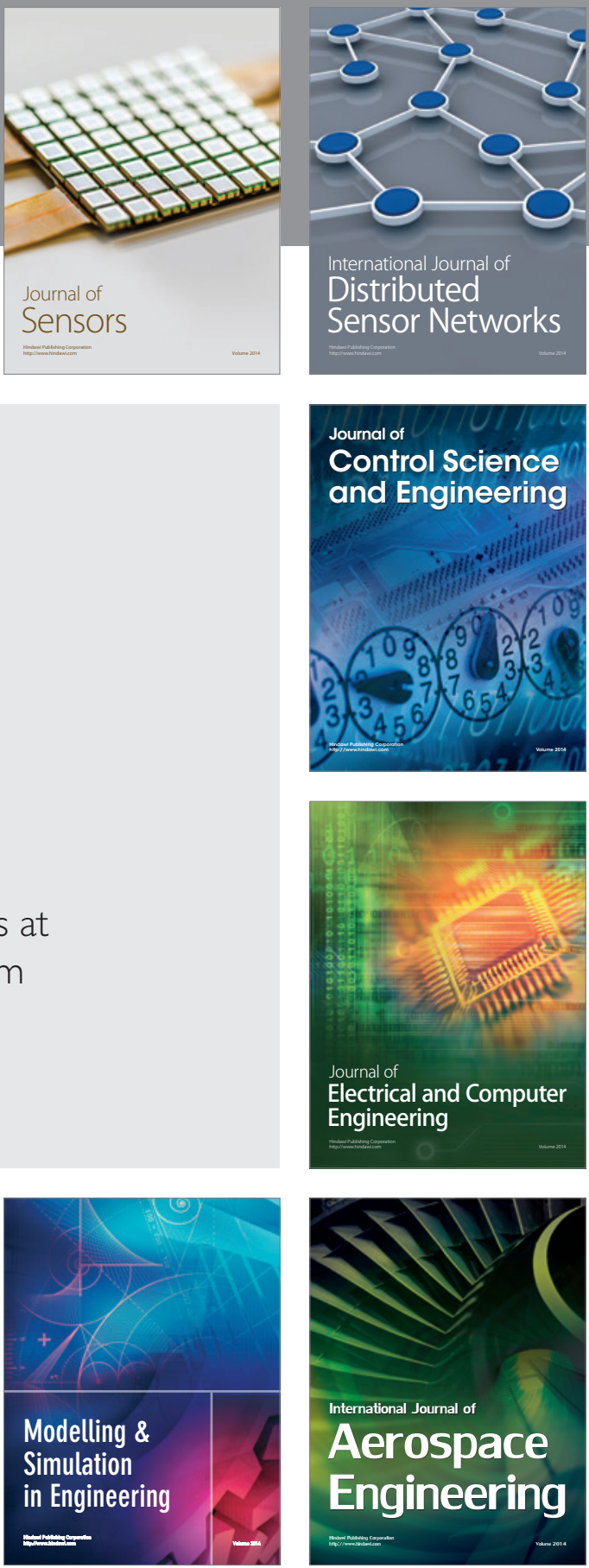

Journal of

Control Science

and Engineering
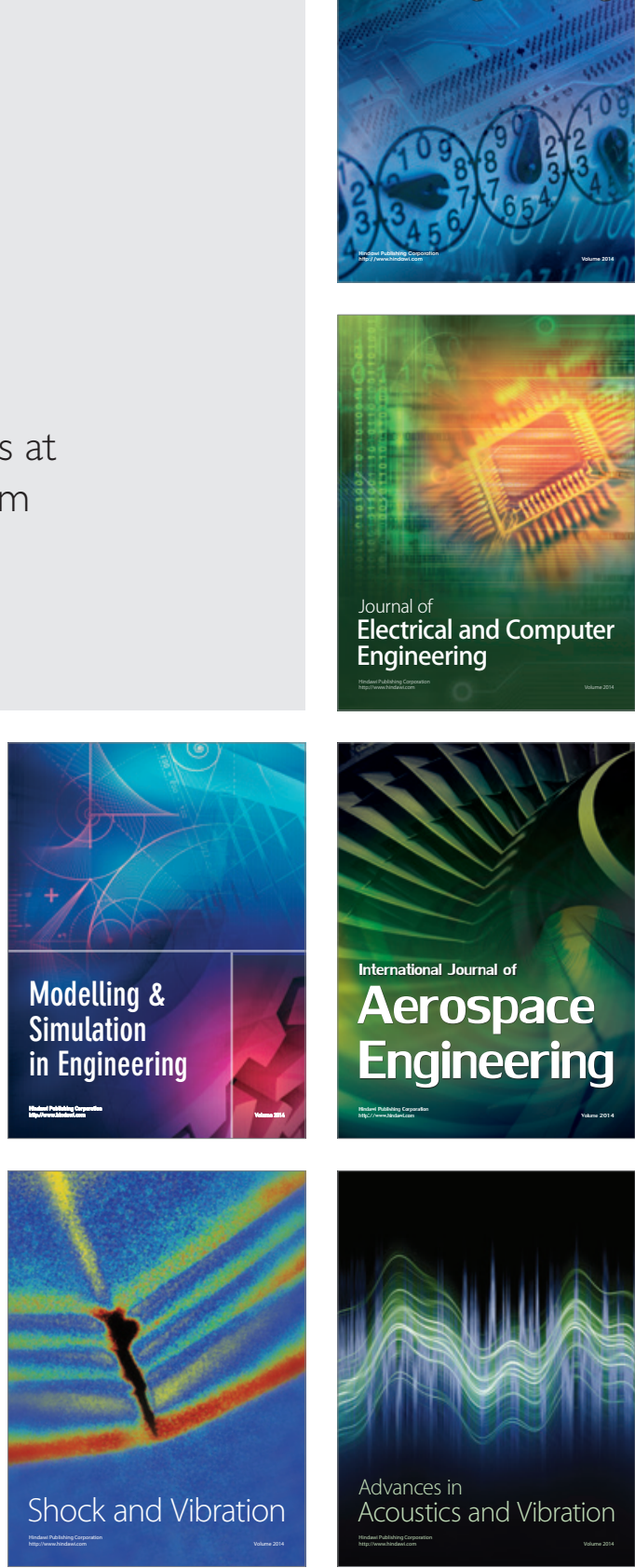